\title{
Problems and Paradoxes of Painting and Perception
}

\author{
Robert Pepperell \\ FOVOLAB, Cardiff Metropolitan University, Cardiff CF5 2YB, UK \\ Received 6 August 2019; accepted 6 August 2019
}

\begin{abstract}
This illustrated essay highlights some conceptual problems that arise when we consider the nature of visual perception and its relationship to art. Science proceeds on the assumption that natural phenomena operate rationally and can be explained rationally. Yet the study of art shows that many ordinary acts of perception, such as looking at a picture, can be paradoxical, logically contradictory and self-referential. I conclude that we must confront these problems if we are to reconcile the scientific approach to explaining visual perception with artistic and philosophical discoveries.
\end{abstract}

\section{Keywords}

Art, perception, paradox, painting

\section{Introduction}

Vision is the most intensively studied of human perceptual faculties. We can measure its optical properties, its psychophysical parameters, and detect neural correlates of visual consciousness with great accuracy. But all this sophisticated analysis tells us little about the strange, even paradoxical nature of visual perception, perhaps the strangest aspect being that we see anything at all.

This illustrated essay highlights some of the perplexing conceptual issues that arise when we think about what perception does, how we perceive artworks, and about artworks that depict what we perceive. As I will show, thinking about these issues presents us with deep logical and conceptual problems.

*E-mail: rpepperell@ cardiffmet.ac.uk 
These problems are rarely discussed in the context of the science of perception. Yet I suggest they cannot be avoided if we are to arrive at a fuller understanding of not only perception itself but of the relationship between ourselves and the world.

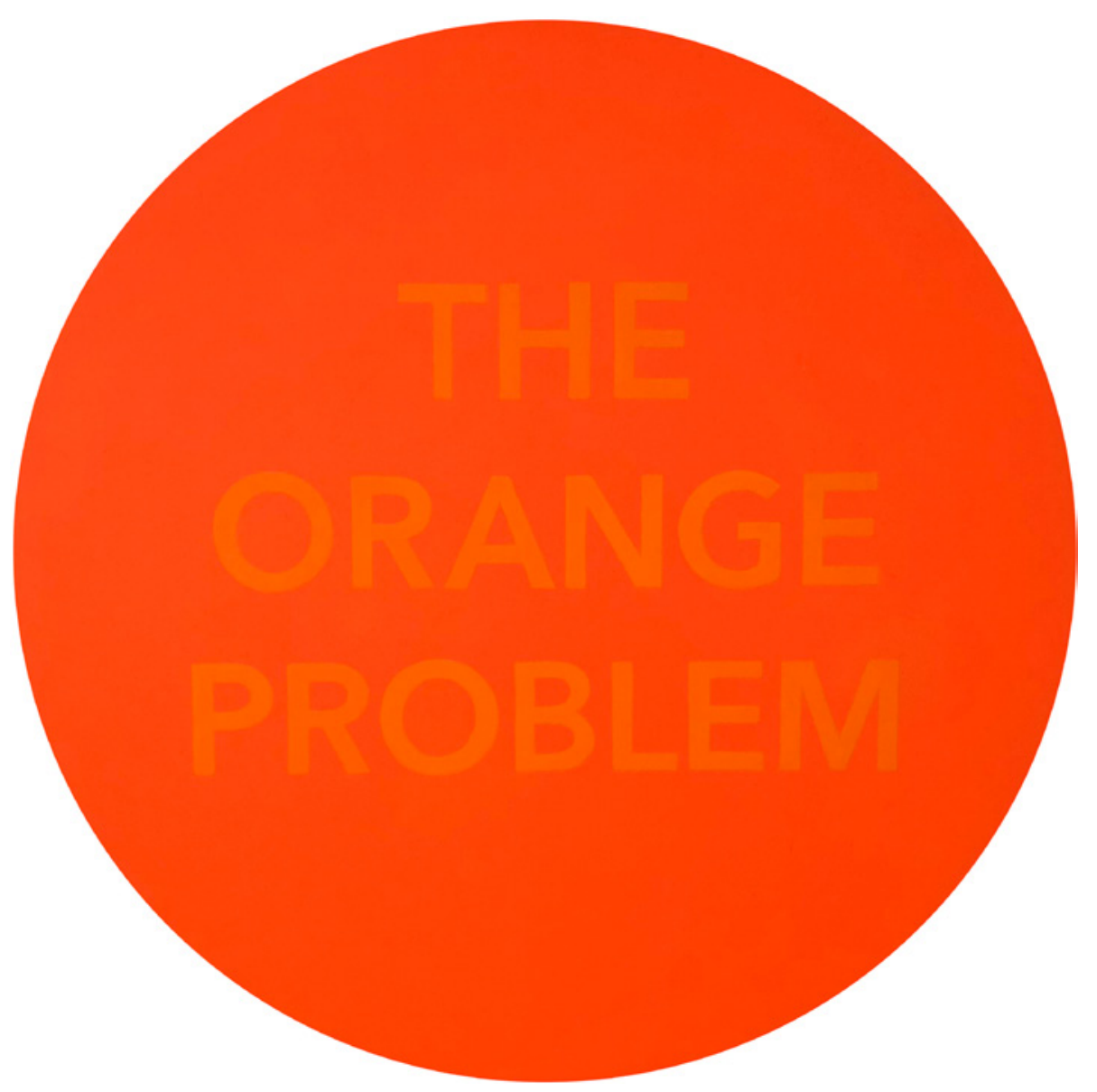

Figure 1. The Orange Problem. Acrylic on round panel, $2018,72 \times 72 \mathrm{~cm}$. $\odot$ Robert Pepperell, 2019. 


\section{The Orange Problem}

I made the painting shown in Fig. 1 to help me think about the nature of colour. The problem (or one of the problems) posed by the painting is 'Where is orange'?

The painting is made with very vivid, almost fluorescent, pigment which mainly reflects light waves in the 635 to 590 nanometre range of the visible spectrum. But neither the paint nor the light it reflects are orange; the painting as a physical object is colourless.

The great breakthrough in physiology made by Johannes Müller's theory of "specific energies" in the early nineteenth century was the realisation that all sensation, whatever its modality or character, is the product of identical electrochemical impulses travelling through the nervous system (Otis, 2007). In 1878, Hermann von Helmholtz expressed one of the startling consequences of this discovery:

The objects in the space around us appear to possess the qualities of our sensations. They appear to be red or green, cold or warm, to have an odour or a taste, and so on. Yet these qualities of sensations belong only to our nervous system and do not extend at all into the space around us. Even when we know this, however, the illusion does not cease, for it is the primary and fundamental truth. (in Cahan, 1995)

But if orange belongs only to our nervous system, then to which part exactly? Cut open a brain, scan it with the best available devices, and you will find no orange. Light from the world, of whatever wavelength, never reaches the visual cortex. So where is orange and where is it perceived? Orange clearly exists; but it is nowhere to be found. And if the perception of orange is an illusion, as Helmholtz claims, then what are we really seeing when we look at the painting? 


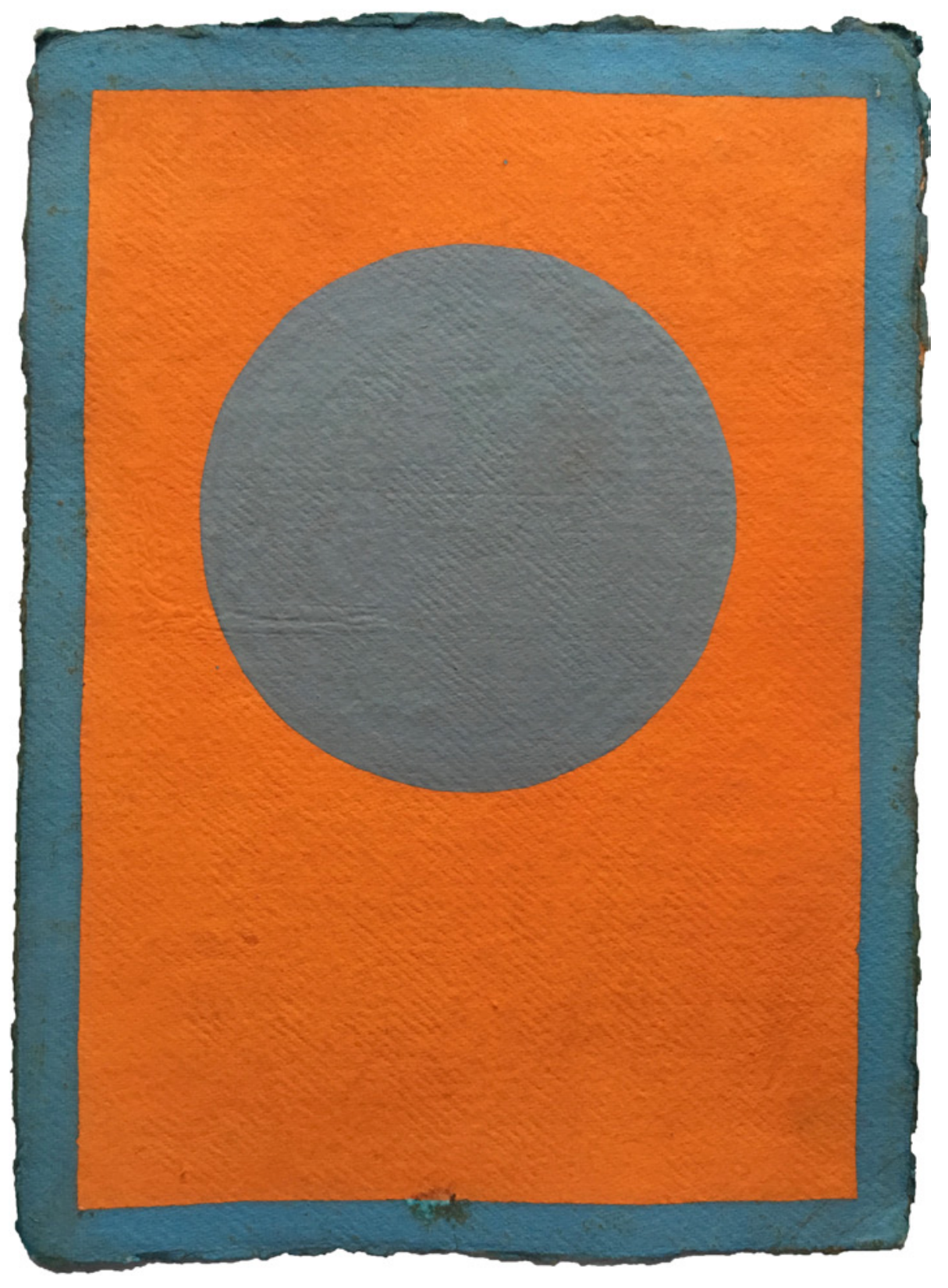

Figure 2. Grey on Orange. Gouache on Indian paper, 2015. $30 \times 20 \mathrm{~cm}$. (C) Robert Pepperell, 2019. 


\section{The Edge of a Circle}

The circle seen in Fig. 2 is perhaps the simplest of forms, yet this apparent simplicity is deceptive. In his book Laws of Form, George Spencer Brown used a circle to denote the "first distinction" that separated something from nothing in his system of algebra (Spencer-Brown, 1979). In doing so he was expressing mathematically what happens whenever we perceive a form in the world: we impose a boundary or difference between one thing and another. To perceive the world is to cut it up.

The human perceptual system works hard to discriminate objects in the environment by enhancing edges or contours. We are highly sensitive to changes in colour or contrast, and virtually insensible to homogeneous or continuous surfaces, or things than don't change. So attuned is the visual system to boundaries that we occasionally see them when they are not there, as with the Kanizsa triangle.

Leonardo da Vinci was puzzled by boundaries. He wondered whether a boundary, like that between the grey circle and the orange background in the painting, belongs to what it encloses or what it excludes. He called a boundary a "line of invisible thickness" (Richter and Bell, 1970), and implied that no such thing could exist. Perhaps it is not that boundaries don't exist but that they are logically contradictory, as Leonardo's phrase suggests. For to perceive an object with a boundary is to perceive both the object itself and what the object is not at the same time.

Those raised in the European philosophical tradition-dominated as it has been by Aristotle's Principle of Non-Contradiction in which something cannot be and not be at the same time-might find such a proposal strange. But the Jain tradition of southern India has long recognised the inherently contradictory nature of object perception. One of Jainism's principal exponents, Satkari Mookerjee, uses the example of a pen on a table to make the point:

The pen is a pen only because it has an individuality which is not the individuality of the table. The pen is perceived as pen and also as not-pen. The not-pen is an indefinite periphery of the pen.

(Mookerjee, 1978)

The edge of the circle is also the edge of everything that is not the circle. 


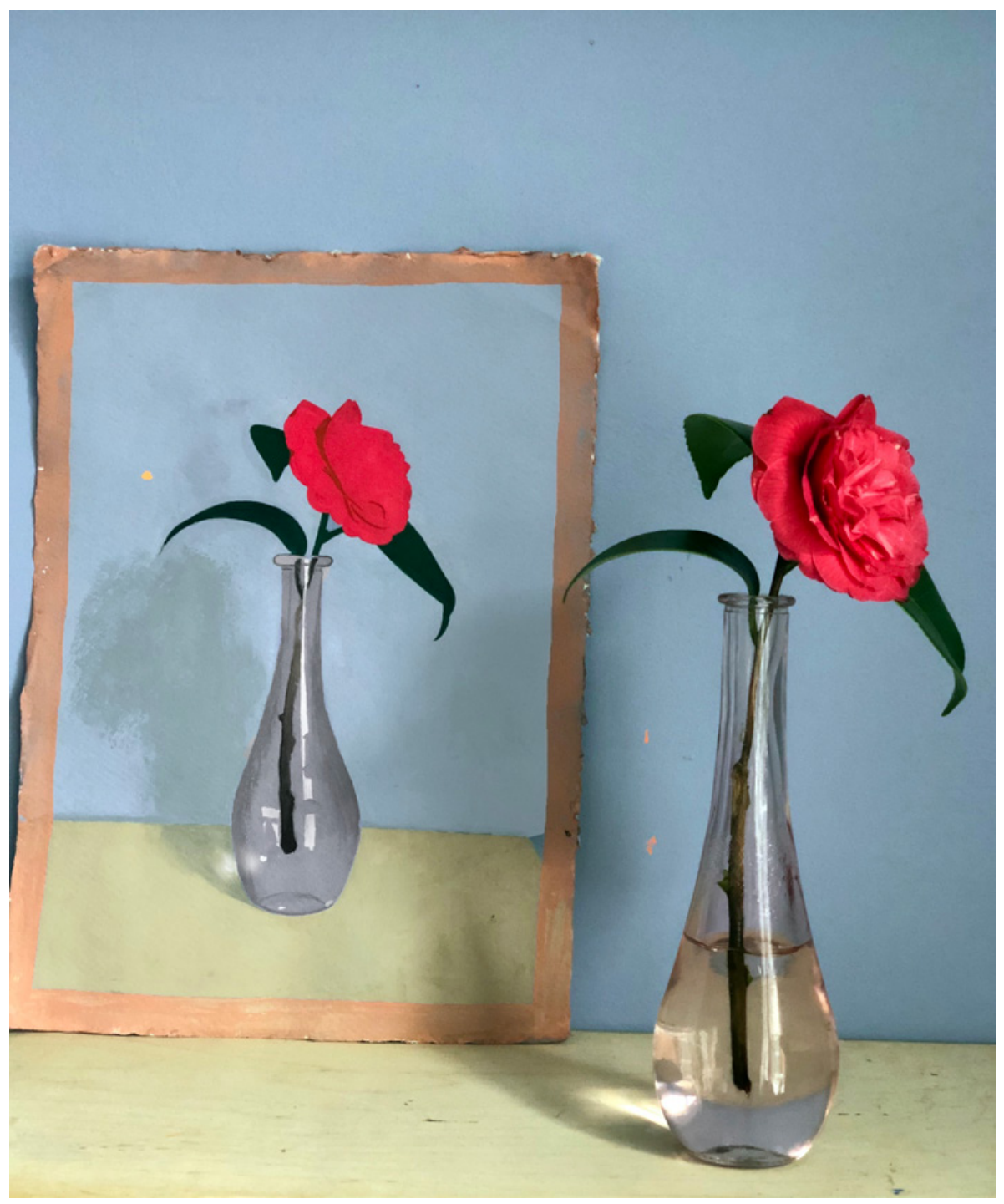

Figure 3. Flower. Gouache on Indian paper, 2019. $30 \times 20 \mathrm{~cm}$. C Robert Pepperell, 2019 . 


\section{The Dichotomy of Depiction}

"Pictures are paradoxical" remarked Richard Gregory (Gregory, 1970). When we look at the painting of the flower in Fig. 3, we see two quite distinct and incompatible things at the same time. We see the flower in the vase and an object made of paint and paper, or of ink or pixels depending on how you are viewing this page. Flowers in vases and stained paper or pixelated screens are quite different kinds of things. Yet when looking at a picture we hardly ever confuse a depicted object with its material substrate. (Exceptions are those rare trompe l'oeil paintings in which we take the represented object to be what it represents.) At the core of each picture, then, lies a contradiction or dichotomy that can never be resolved if we are to perceive it as a picture. The philosopher of art Richard Wollheim called this the "two-foldedness" of pictures (Wollheim, 1980).

Many people assume, and some argue, that visual perception is veridicalthat the job of vision is to give us an accurate and unambiguous account of what is really before us in the world. But pictures undermine that idea. For there is nothing veridical about seeing flowers that are not there, or confusing paint, ink or pixels with plants and glass. Numerous visual illusions-such as the Kanisza triangle, the Hermann grid, and the Ebbinghaus discs-testify to the fact that we do not always accurately perceive what is before us. Nor does veridicalism allow for the great diversity of visual experiences that people with different knowledge, training, experience, cultural backgrounds, and expectations can have when they see the same things in different ways. As James Gibson (1968) pointed out, an expert can be sensitive to things that a novice is oblivious to.

So, if visual perception is not veridical, is it arbitrary? Do we just make up what we see regardless of what is actually there? To some extent, but not entirely. Visual perception, as several people have suggested, is a "controlled hallucination" (Koenderink and van Doorn, 2008). The objective world has an influence on what we see, but so do prior knowledge, experience and expectations. And we only see what our particular biological apparatus allows; many creatures see far more than us, many far less (Land and Nilsson, 2012).

The dichotomous nature of pictures tells us that visual perception is nonveridical. 


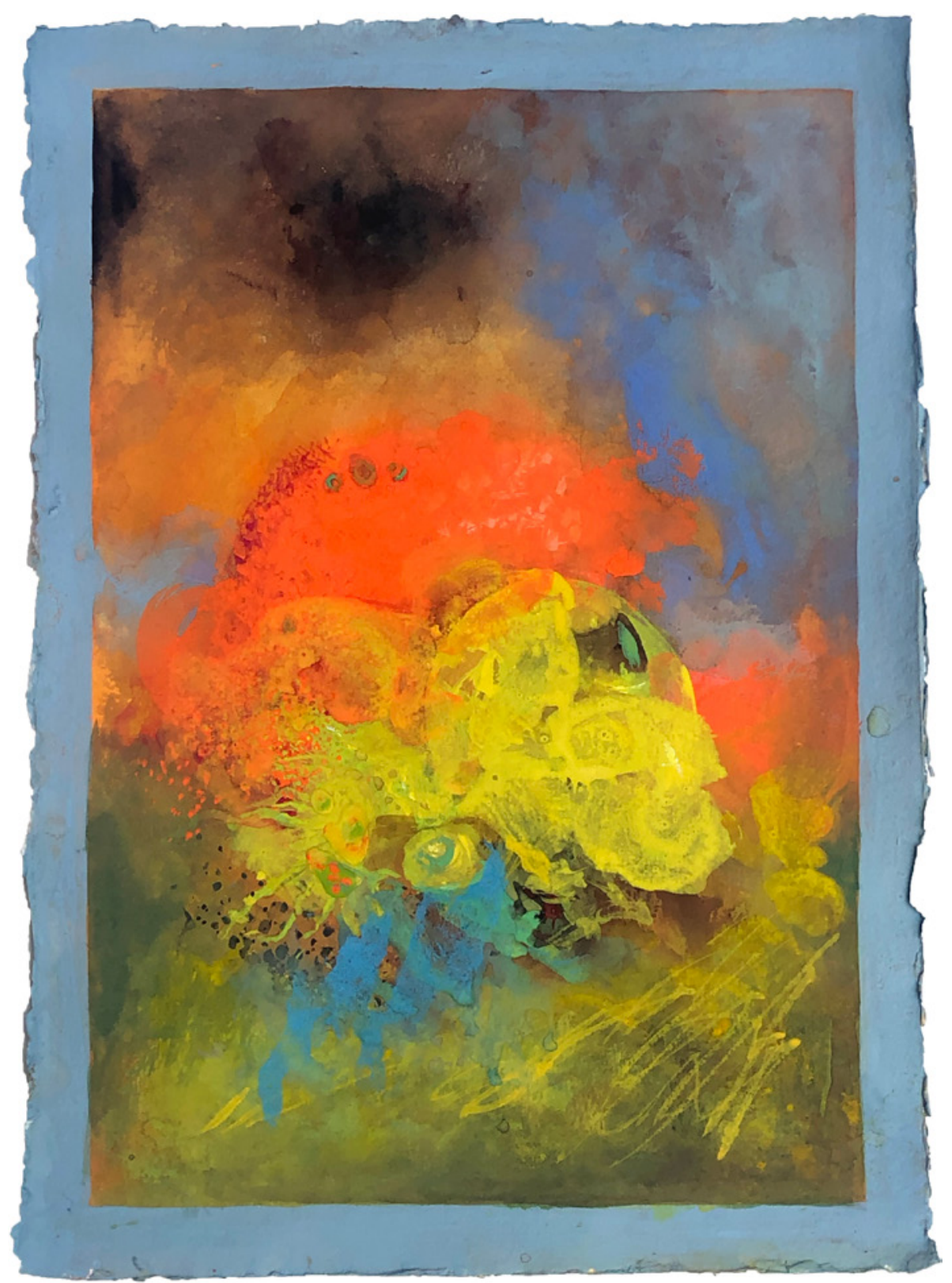

Figure 4. On the Edge. Gouache on Indian paper, 2019. $30 \times 20 \mathrm{~cm}$. (C) Robert Pepperell, 2019. 


\section{The Location of Objects}

You may be unsure of what this painting depicts. It is one of a series of works that are semantically indeterminate. Indeterminate images resist immediate classification. They can be slightly frustrating to view because our visual system is optimised for rapid and effective object recognition; we like to be sure what it is we see, and quickly.

In the absence of an immediate interpretation you might find yourself scrolling through options in your mind, searching for objects that 'fit' the clues. (Is it a sea creature or some kind of cosmic storm?) If so, you are experiencing at a slow pace what usually occurs so quickly that you never notice it. Your visual system is working to match its input with your prior knowledge to arrive at the best guess of what is being seen. Even before this matching can occur, an enormous amount of processing has already been done by the visual system, in the retinae and in the cortex, to build up a perceptible image from 'primitive' elements like edges, corners, and contrasts of colour and brightness.

The fact that vision has to do all this work before we can recognise an object shows us that the objects we perceive are not just 'there'. They have to be created by our nervous system, as Helmholtz noted, in order to exist for us. But again, cut open a brain, scan your neurons, and you will find no sea creatures or cosmic storms, or even pictures of such things. So where do objects exist?

Hard as it may be for us to accept, the world is indeterminate prior to perception. We carve the world up into objects according to our needs. But it can be carved up in an infinite number of ways depending on what you expect to see or how your perceptual system works. Bees and bats perceive the world quite differently from us, although we all inhabit the same world.

Indeterminate images, then, are arguably more realistic than recognisable images because they present the world to us in a way that is closer to how it objectively is (void of objects) rather than to how we subjectively perceive it (object-full). 


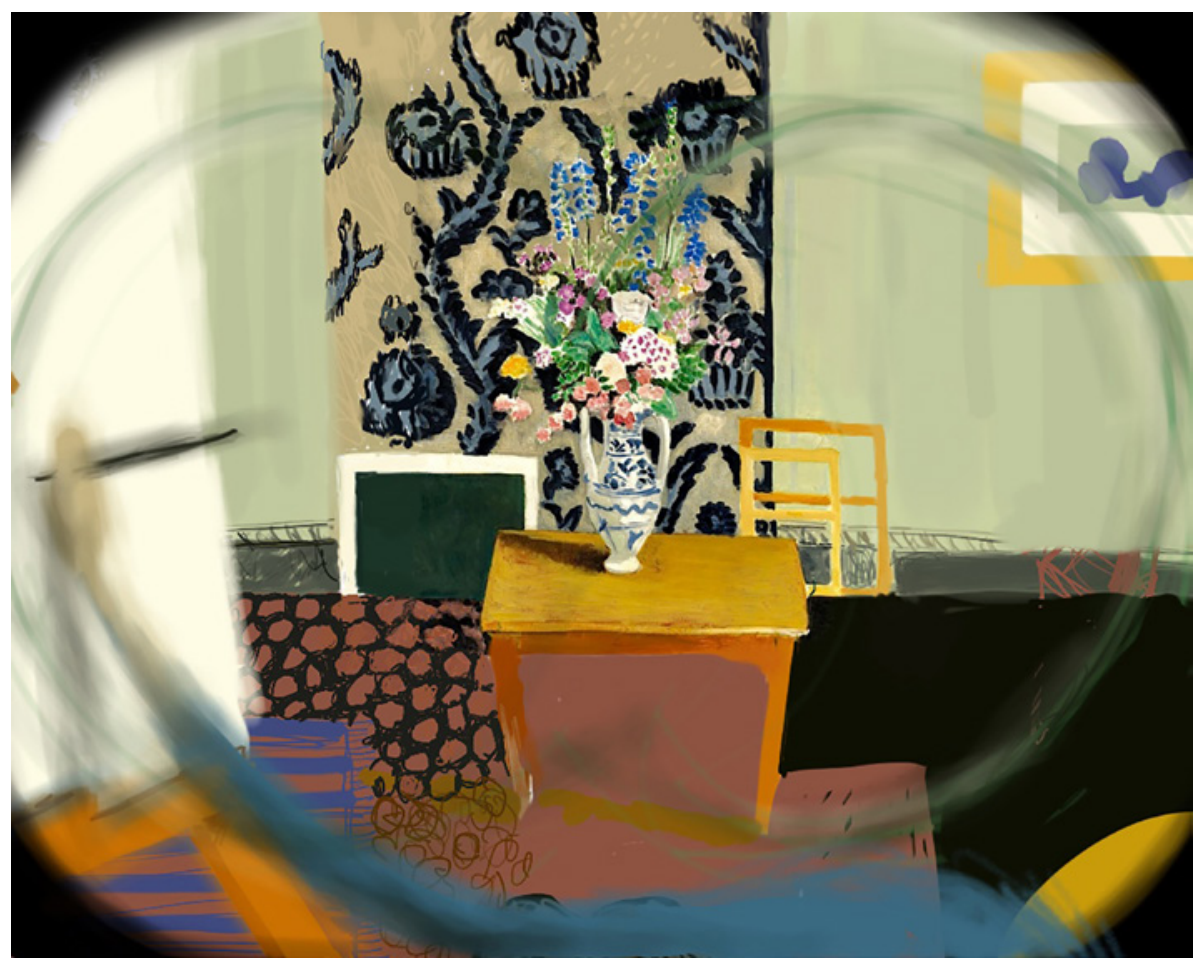

Figure 5. Looking as Matisse (After Still Life with Flowers by Matisse). iPad drawing, 2017. (C) Robert Pepperell, 2019. 


\section{Looking-at and Looking-as}

The iPad drawing in Fig. 5 is based on a painting of a vase of flowers made by Henri Matisse. Matisse said: "I don't paint objects. I paint the only differences between objects" (Delectorskaya et al., 1996). This could equally apply to visual perception: "I don't see objects. I see only the differences between objects."

Matisse was an acute observer of his own visual sensations. This is evident in many of his paintings and drawings where he captures often overlooked features of vision such as the curvature of peripheral space or his own hands holding the pen as he draws a model. In this drawing I have extended the visual space shown in Matisse's original painting, which covered only the vase of flowers and a small region of the space around it, in order to show the entirety of what he would have seen when seated at his easel and studying the flowers. I have added extra wall and floor space, his arm holding the brush, the easel, the painting he is painting, and the fuzzy outlines of his steel-rimmed spectacles.

This drawing invites you to look differently. Rather than looking at the drawing, as you might normally do, try to look into the space it depicts, as though you were looking through the artist's eyes. (This sometimes works better with one eye closed.) You may start to feel a heightened sense of spatial presence and depth not normally associated with flat pictures, and a slightly uncanny sensation of looking from inside someone else's head towards the world 'out there'.

I call this 'looking-as' rather than 'looking-at', in a nod to the distinction between 'seeing-in' and 'seeing-as' made by the philosopher Richard Wollheim in his theory of pictorial perception (Wollheim, 1980). By 'looking-as' I mean that you are looking as the person whose visual perception is being depicted looked, rather than at a surface with a depiction on it. Compared to the conventional mode of viewing this is a more embodied, subjective way of viewing a picture that, I think, better accords with what many artists are trying to do when they strive to capture their own visual experience of the world in paint.

By looking as Matisse looked you look at what he saw, and what he saw was as much in his mind as it was 'out there'. 


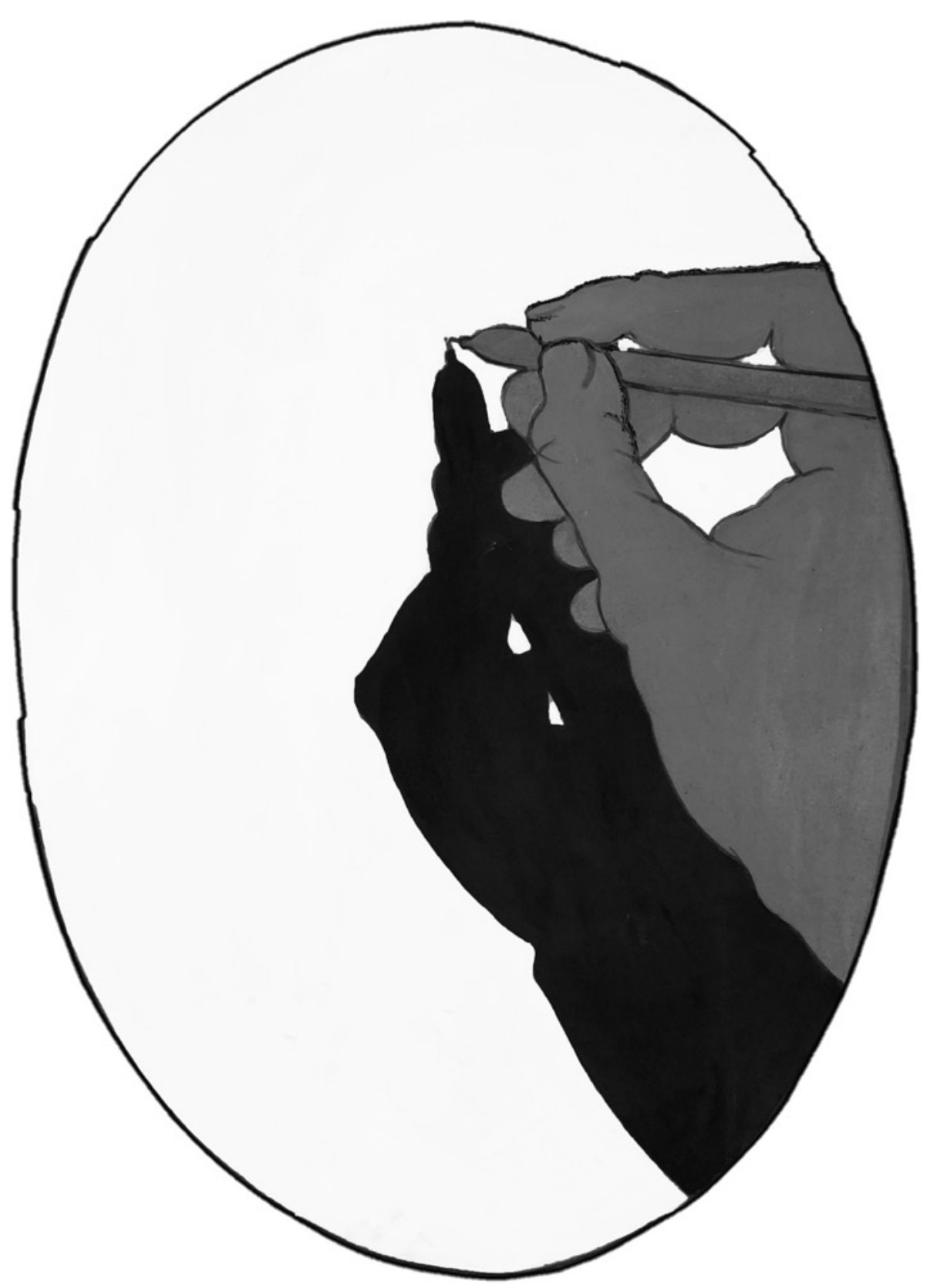

Figure 6. Drawing drawing. Gouache on paper, $2011.40 \times 30 \mathrm{~cm}$. @ Robert Pepperell, 2019 . 


\section{Self-Reference}

The drawing reproduced in Fig. 6 shows my hand in the process of drawing the drawing you see. Artists are fond of pictorial self-reference, which occurs when the picture refers to the means of its own production. Perhaps the most famous example is the magnificent Las Meninas by Velasquez. But there are many others in art history, from the startling trompe l'oeil illusions of Cornelius Gijsbrechts to the surrealist conundrums of René Magritte and the perceptual teasers of M.C. Escher.

One reason that self-referential pictures are fascinating is that they alert us to the inherent paradoxicality of all depictions. Paintings and drawings are real enough as objects, yet there is nothing real about the objects that appear in them, however realistically depicted.

Here you are looking at a drawing of a hand (in reproduction) in which you read certain lines and patches as skin, a pencil, a shadow, and paper. We might say that these lines and patches, which are present, evoke things that are absent since there is no hand or shadow there. Yet the same is not true of the pencil lead with which I made the drawing. This can be seen at the tip of the pencil both representing a pencil lead and being actual pencil lead at the same time. The paper on which I am drawing is both a representation of paper and actual paper. These objects are simultaneously absent and present.

This might be dismissed as trivial were it not for the fact that it exposes a remarkable property of our perceptual faculties. For if we run into logical problems conceiving of how something might be present and absent simultaneously, we have no trouble perceiving it. Perception seems to take contradiction in its stride.

Finally, we must accept the bizarre but nevertheless "primary and fundamental truth", as Helmholtz put it, that all perception is self-referential. When you or I look at the world we never see the world itself, contrary to appearances, but our own perceptual construction of it. Just as the drawing shows my hand in the act of drawing itself, so vision shows us in the act of perceiving ourselves. 


\section{Conclusion}

I have tried to show with examples of my own paintings and drawings that art can address deep and complex problems in perception, and that it can contribute to our understanding of these problems, even if only by exposing them more vividly. My view, as expressed here, is that perceiving the world and perceiving depictions of the world are highly perplexing acts with implications that test the bounds of rationality. Visual perception, it seems, can be paradoxical, contradictory and self-referential; it is a far stranger beast than we are often led to believe.

It would be tempting to side-step these conceptual problems. We may feel inclined to dismiss them as logical quirks of little relevance to the serious science of perception, or worse still as symptoms of artistic eccentricity. But if we are to make progress in understanding perception, and the intimate bond between art and perception, then I believe we have no choice but to confront them. We may even find-as I suspect we will—that doing so can help us meet the scientific and intellectual challenges we face in explaining how we see.

\section{References}

Cahan D. (1995). Hermann Von Helmholtz: Science and Culture, Chicago University Press, Chicago, IL, USA.

Delectorskaya L., Matisse H. and Monod-Fontaine I. (1996). Henri Matisse, contre Vents at Mares: Peintures et Livres Illustrés de 1939 a 1943, Editions Irus et Vincent.

Gibson J. (1968). The theory of information pickup, in Contemporary Theory and Research in Visual Perception, R.N. Haber (Ed.), pp. 662-678, Holt, Rheinhart and Winston, New York, NY, USA.

Gregory R. (1970). The Intelligent Eye, McGraw-Hill Book Company, New York, NY, USA.

Koenderink J. and van Doorn A. (2008). The Structure of Visual Spaces. J Math Imaging Vis. 31, 171-187.

Land M. and Nilsson D.E. (2012). Animal Eyes, Oxford University Press, Oxford, UK.

Mookerjee S. (1978). The Jaina Philosophy of Non-Absolutism, Motilal Banarsidass, Delhi, India.

Otis L. (2007). Müller's Lab, Oxford University Press, Oxford, UK.

Richter J. P. and Bell R. (1970). The Notebooks of Leonardo da Vinci, Dover, New York, NY, USA.

Spencer-Brown G. (1979). Laws of Form, E.P. Dutton, New York, NYU, USA.

Wollheim R. (1980). Art and Its Objects, Cambridge University Press, Cambridge, UK. 